\title{
Sliding Synchronous Control of Random Second Order System with a Known Control Direction
}

\author{
Xinyu Wang \\ Institute of Science and Technology for opto-electronic information, Yantai \\ University, Yantai \\ wangxinyu1024@126.com
}

\begin{abstract}
A kind of synchronization and tracking control method which in mostly used in synchronization of communication systems in the past, is firstly proposed for a kind of random second order system with single known control directions. System uncertainties are coped by constructing synchronous system with different structure. Synchronization law is designed to realize the synchronization between original system and auxiliary system. Then the state of auxiliary system can track the objective system. After that, a sliding mode tracking control is designed for the auxiliary system and its state can tracking the desired value. Then the state of uncertain second order system can also tracking the desired value and the control objective is realized. At last, detailed numerical simulation of PID control and sliding synchronous control are done to testify the rightness and effectiveness of the proposed method.
\end{abstract}

Keyword: Synchronization; Sliding mode control; Second order system; Stability; Lyapunov function

\section{Introduction}

Synchronization was firstly observed by Huygens in 1673 when he researched on coupled pendulums. In fact, there are many kinds of synchronization phenomenon [1-10] in coupled systems with interaction. For example, fireflies blink at the same time although each of them is different from each other. They can realize synchronization by some kinds of interaction. And we realized that synchronization not only exists widely in all real life but also it is very useful such as synchronization in secure communication. But also some synchronizations are bad and should be avoided by human beings such as information jam of internet and step synchronization of soldiers when they walk across a bridge.

Now researches of synchronization with control theory mainly focus on the following two parts. One is synchronization and its application in communication systems. There are many papers which studied adaptive synchronization or robust synchronization of chaotic systems with different structure, or with model uncertainties and unknown parameters. For example, Jianping Yan [10] realized synchronization of three chaotic systems with similar structure by using Lyapunov function method in 2005. E. M. Elabbasy [11] studied synchronization of Lu chaotic system with same structure but unknown parameters. Adaptive law was designed to estimate the unknown parameters and feedback controller was designed for synchronization by adopting Lyapunov stability theorem. M. T Yassne, H. NAgazi and Ming-chung Ho [12-14] used nonlinear feedback method to cope the nonlinear part of error system. And Lyapunov function, Hurwitzs equation and poles assignment methods were adopted to solve the conditions of synchronization. But this method can only be used for given special chaotic system, and it is difficult to be applied to a general nonlinear chaotic system. 
Another part is focus on the synchronization of networks with complex dynamics. Those researches are very effective for networks with known structure and known parameter or known coupled functions. And it is more difficult to realize synchronization of two networks with unknown parameters or network structure. Some researchers tried to use adaptive method or robust method to achieve synchronization. So there are many papers about robust synchronization or adaptive synchronization of complex networks.

With the study of references above, it is obvious that the system uncertainties are the main difficult problem of both above two kinds of research work about synchronization [15-19]. Many kinds of uncertainties are studied and the most serious situation is those systems with nonlinear uncertainties which can not be described by bounded linear functions. So generally speaking, the main problem of above synchronization can be transferred to be a control problem. Also system with uncertainties is the main problem that designers should consider and solve.

There is another common point of above two kinds of researches which is easy to be neglected by readers is that all above synchronization problems can be defined as a controller design and stability analysis problem of high order system with multi-inputs[20-26]. In fact, there are only a few papers tried to use single input to realize synchronization of two high order systems. It is worthy pointing out that it is more difficult to realize synchronization of high order system with single input than to realize it with multi-inputs. Also, if we consider the situation that some states of system can not be measured, then the question can be more difficult.

In this paper, a new kind of synchronization control method is firstly defined and proposed. synchronization is used to control uncertain high order system with single input, so it is difficult for some systems. This synchronization control method is the same as PID control, adaptive control and variable structure control and other general control method that can be used for a common control system. Also, this method can be completely applied in above chaotic synchronization problem and complex networks.

A kind of novel auxiliary synchronous system is constructed to cope the uncertainties of high order systems. This novel control structure is similar but different to referenced model control method. The original system is uncertain and the auxiliary system is certain. The synchronization of above two systems is realized by constructing multi-virtual synchronous controls which are also designed to cope uncertainties of original system. Those multi-virtual synchronous controls are decoupling for high order system so it is easy to design. And the real control law is designed according to an auxiliary system without uncertainties by using sliding mode control method. So it is called sliding mode synchronous control method. To make it simple and show the main principle of the new control method, a kind of random second order system is taken for an example and the whole design procedure and stability analysis are given in this paper. At last, detailed simulation and comparison are done to show the rightness of the new method.

\section{Problem Description}

Second order system with single control direction is a special simple situation of second order system research. The control direction is defined as the coefficient of input $u$. And the model is defined as

$\dot{x}_{1}=a_{11} x_{1}+a_{12} x_{2}$

$\dot{x}_{2}=a_{21} x_{1}+a_{22} x_{2}+u$

Where $x_{i}(i=1,2)$ are system states, $a_{i}(i=1,2)$ are parameters of control system. 
The control objective is to design a control law $u=f(x)$ such that the system stat $x_{1}$ can track the desired value $x_{1}^{d}$. Without loss of generality, assume the desired value $x_{1}$ as $x_{1}^{d}=1$.

\section{PID Control Law Design}

The structure of PID control is showed as following Figure 1. The system is constructed by PID controller and control object. And the object is controller by PID controller which is consisted by proportional item, differential item and integral item.

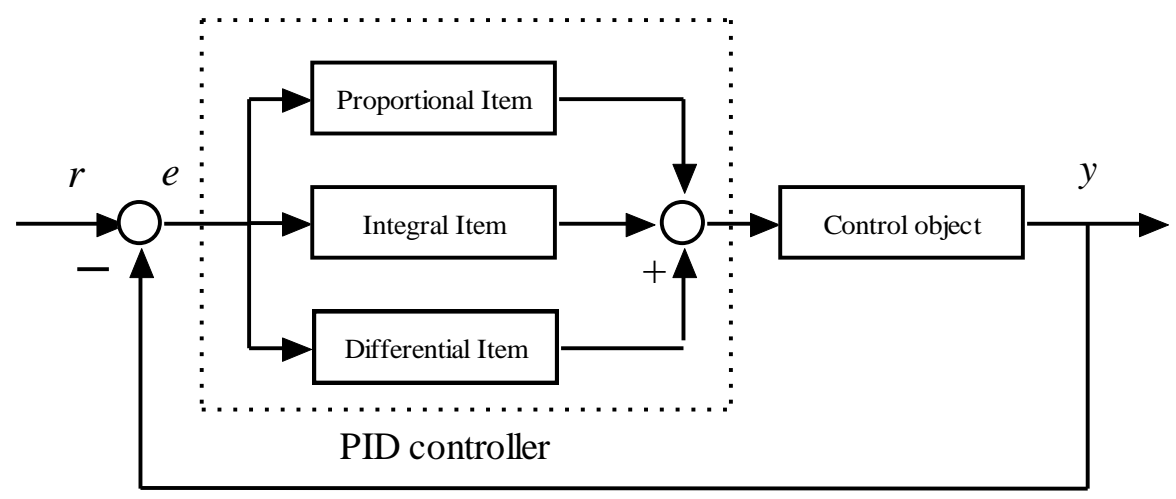

Figure 1. Structure of PID Control System

PID controller is a kind of linear controller, it is composed by the error signal defined by the difference between the desired value $x_{1}^{d}$ and output of system $x_{1}$ as follows:

$e(t)=x_{1}-x_{1}^{d}$

The PID control law is designed as

$u(t)=k_{p}\left(e(t)+\frac{1}{T_{I}} \int_{0}^{t} e(t) d t+\frac{T_{D} e(t)}{d t}\right)$

It can be written as a transfer function as

$$
G(s)=\frac{U(s)}{E(s)}=k_{p}\left(1+\frac{1}{T_{I} s}+T_{D} s\right)
$$

Where $k_{p}$ is the coefficient of proportional item and $T_{I}$ is the coefficient of integral item and $T_{D}$ is the coefficient of differential item.

\section{Sliding Mode Control Law Design}

Assumption 1: $a_{12}$ is not zero and $a_{12}>0$.

Assumption 2: desired value $x_{1}^{d}$ is a constant so $\dot{x}_{1}^{d}$ is zero.

Sliding mode method is a kind of synthesized method and it is very simple and easy for designers. There are two main parts for designing a sliding mode control. First part is to design a sliding mode $s(x)$ according to the desire of performance. Second part is to solve the sliding mode control law according to the sliding mode.

Define the error variable $e=x_{1}-x_{1}^{d}$ then

$$
\dot{e}=\dot{x}_{1}=a_{11} x_{1}+a_{12} x_{2}
$$

The second order derivative of error can be solved as 


$$
\begin{aligned}
& \ddot{e}=a_{11} \dot{x}_{1}+a_{12} \dot{x}_{2} \\
& =a_{11}^{2} x_{1}+a_{11} a_{12} x_{2}+a_{21} a_{12} x_{1}+a_{12} a_{22} x_{2}+a_{12} u
\end{aligned}
$$

And define the sliding mode as

$$
s=\dot{e}+c_{1} e+c_{2} \int e d t
$$

It is necessary to set $c_{1}>0, c_{2}>0$ such that the sliding mode surface is stable. It means that when $s=0$ then the differential equation is stable.

Solve the derivative of the differential equation, it holds:

$$
\begin{aligned}
& \dot{s}=\ddot{e}+c_{1} \dot{e}+c_{2} e \\
& =\left(a_{11}^{2}+a_{21} a_{12}+a_{11} c_{1}\right) x_{1}+\left(a_{11} a_{12}+a_{12} a_{22}+a_{12} c_{1}\right) x_{2}+c_{2} e+a_{12} u
\end{aligned}
$$

Define

$$
\begin{aligned}
& l_{1}=a_{11}^{2}+a_{21} a_{12}+a_{11} c_{1} \\
& l_{2}=a_{11} a_{12}+a_{12} a_{22}+a_{12} c_{1} \\
& l_{3}=c_{2}
\end{aligned}
$$

Design

$$
u=\left(-\hat{l}_{1} x_{1}-\hat{l}_{2} x_{2}-\hat{l}_{3} e-k_{1} s-k_{2} \operatorname{sgn}(s)-k_{3} \int s d t\right) / a_{12}
$$

Define the turning law as follows

$$
\begin{aligned}
& \dot{\hat{l}}_{1}=\Gamma_{1} \alpha s \\
& \dot{\hat{l}}_{2}=\Gamma_{2} \omega_{z} s \\
& \dot{\hat{l}}_{3}=\Gamma_{3} e s
\end{aligned}
$$

It is easy to prove that the system is stable.

\section{Sliding Mode Synchronization Controller Design}

Since there are many kinds of second order systems, it is very important that the same controller can be applied to many different second order systems. So it is meaningful to design a universal synchronous system that it is effective to all kinds of second order system. So a kind of synchronous control method is proposed to solve this problem and it is a kind of universal method with structures as follows:

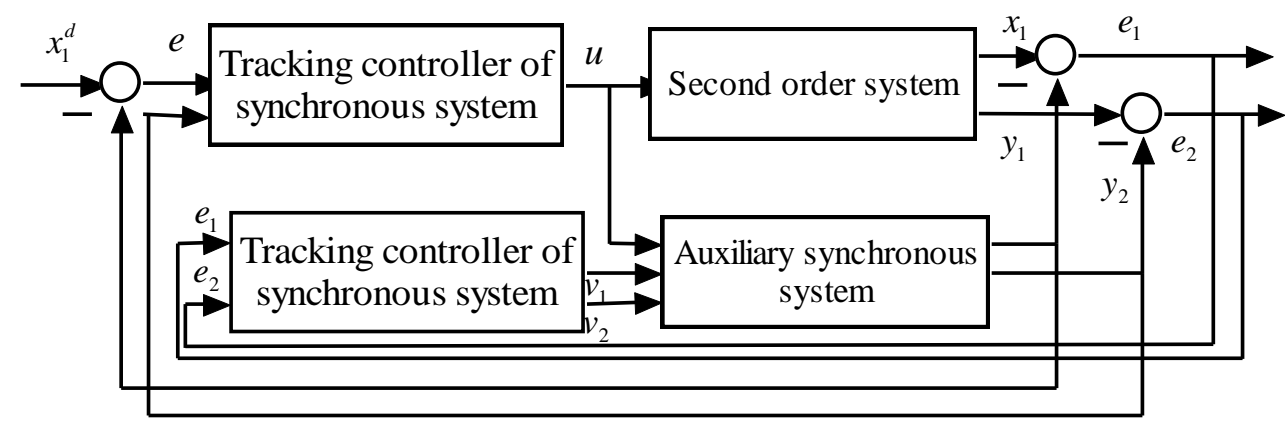

Figure 2. Structure of Synchronous Control

So the auxiliary synchronous system can be constructed as

$$
\begin{aligned}
& \dot{y}_{1}=b y_{2}+v_{1} \\
& \dot{y}_{2}=u+v_{2}
\end{aligned}
$$


where $v_{i}(i=1,2)$ are control such that the synchronization between two systems can be realized. $b$ is a system parameter.

The objective of auxiliary synchronous system is to design a synchronous law $v_{i}(i=1,2)$ and parameter $b$ such that the state of synchronous system can track the state of original system. It means that $y_{i} \rightarrow x_{i}(i=1,2)$.

Define a synchronous error as $e_{i}=y_{i}-x_{i}(i=1,2)$, then the error system can be defined as

$$
\begin{aligned}
& \dot{e}_{1}=\dot{y}_{1}-\dot{x}_{1} \\
& \dot{e}_{2}=\dot{y}_{2}-\dot{x}_{2}
\end{aligned}
$$

Then it can be written as

$$
\begin{aligned}
& \dot{e}_{1}=b y_{2}-a_{11} x_{1}-a_{12} x_{2}+v_{1} \\
& \dot{e}_{2}=-a_{21} x_{1}-a_{22} x_{2}+v_{2}
\end{aligned}
$$

Then the problem is that how to design a synchronous control law $v_{i}(i=1,2)$ and choose a parameter $b$ such that the error $e_{i}$ can converged to zero.

Design a synchronous control law as

$$
v_{i}=-k_{p i} e_{i}-k_{s i} \int e_{i} d t
$$

Choose the Lyapunov function as

$$
V=\frac{1}{2} e_{1}^{2}+\frac{1}{2} e_{2}^{2}
$$

Solve its derivative as

$$
\begin{aligned}
\dot{V}= & e_{1} \dot{e}_{1}+e_{2} \dot{e}_{2} \\
= & -k_{p 1} e_{1}^{2}-k_{p 2} e_{2}^{2}-k_{s 1} e_{1} \int e_{1} d t-k_{s 2} e_{2} \int e_{2} d t \\
& +e_{1}\left(b y_{2}-a_{11} x_{1}-a_{12} x_{2}\right)+e_{2}\left(-a_{21} x_{1}-a_{22} x_{2}\right)
\end{aligned}
$$

Choose $k_{p 1}$ and $k_{p 2}$ to be big enough positive numbers, then the error system can be proved to be stable according to the Lyapunov stability theorem.

Define a new error variable as:

$$
e=y_{1}-y_{1}^{d}
$$

Choose a sliding mode surface composed by three items such as error item, integration of error and derivative of error as follows:

$$
s=c e+d \int e d t+\dot{e}
$$

Solve its derivative as

$$
\begin{aligned}
& \dot{s}=\ddot{e}+c \dot{e}+d e=b c y_{2}+c v_{1}+d e+b u+b v_{2}+\dot{v}_{1} \\
& \text { If } \dot{s}=0, \text { it holds } \\
& \ddot{e}+c \dot{e}+d e=0
\end{aligned}
$$

So it is necessary for the system (29) have two negative roots to make the sliding surface stable. Hence parameters are chosen as $c=2, d=5$.

And design the control law as

$$
u=\frac{1}{b}\left(-b c y_{2}-c v_{1}-d e-b v_{2}-\dot{v}_{1}-k_{1} \operatorname{sgn}(s)-k_{2} s-k_{3} s /(|s|+0.2)\right)
$$

Where parameters are chosen as $c=2, d=5, k_{1}=0, k_{2}=10, k_{3}=1, b=1$.

It is easy to prove that the system is stable by choose a Lyapuov function as $V=s^{2} / 2$. Then the control law design is complete and simulations are done below to testify the rightness of above design. 


\section{Numerical Simulation}

Choose a second order system as

$\dot{x}=A x+b u$

Where $A=\left[\begin{array}{ll}a_{11} & a_{12} \\ a_{21} & a_{22}\end{array}\right], b=\left[\begin{array}{l}0 \\ 1\end{array}\right]$.

\subsection{Simulation of PID Control}

Choose parameters of matrix $A$ to be rand numbers between (-10 10). For example, choose parameters as follows:

$$
a_{11}=8.7 ; a_{12}=-3.6 ; a_{21}=-9.3 ; a_{22}=7
$$

And design PID control parameters as $k_{p}=-10, k_{i}=-5, k_{d}=-1$ for above system and the simulation result are shown as Figure 3.

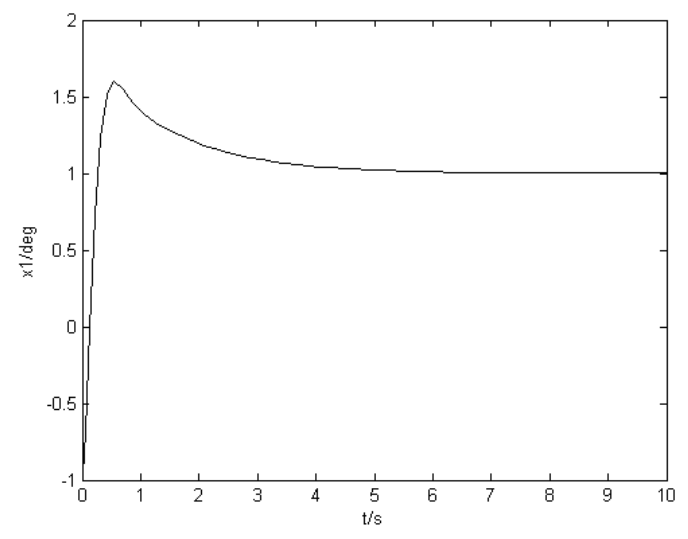

Figure 3. Simulation Result of PID Control

Use above control parameters and choose six group of rand numbers for matrix A, the control result are shown as below figures.

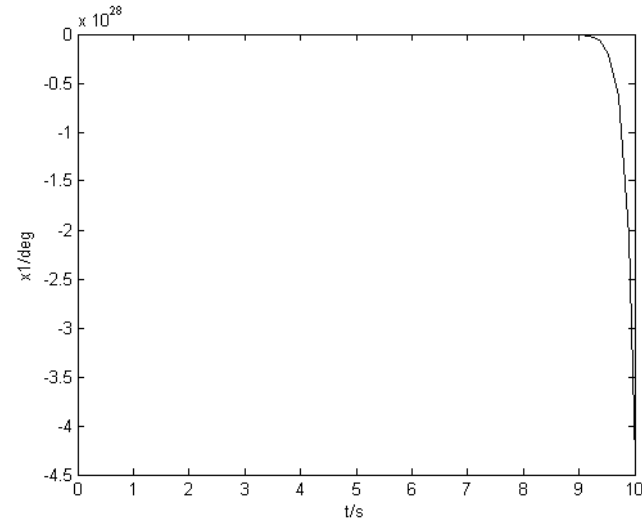

Figure 4. Result of First Group

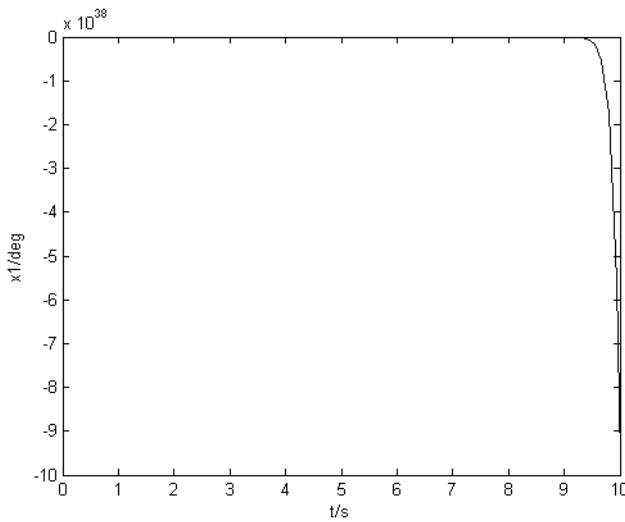

Figure 5. Result of Second Group 


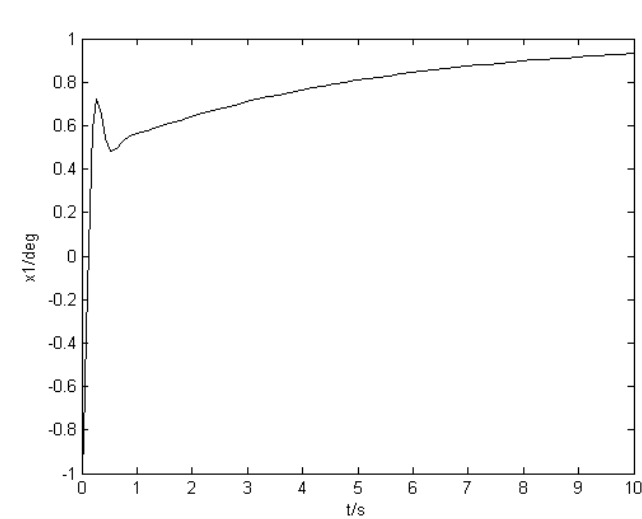

Figure 6. Result of Third Group

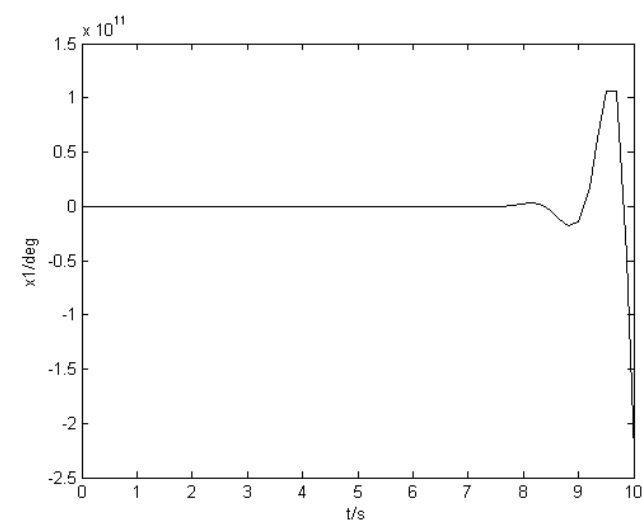

Figure 8. Result of Fifth Group

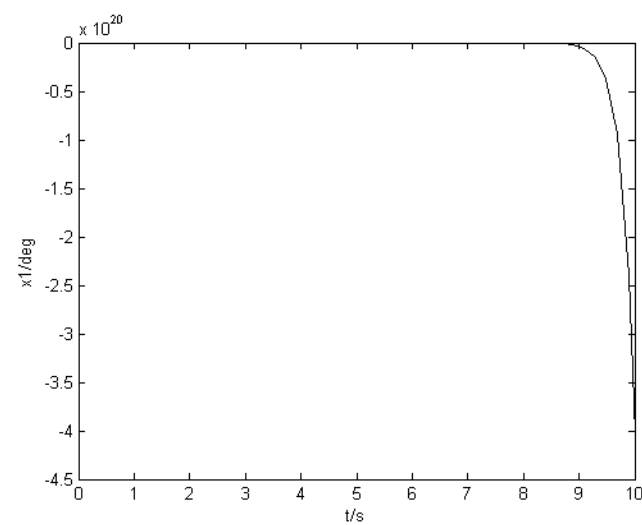

Figure 7. Result of Fourth Group

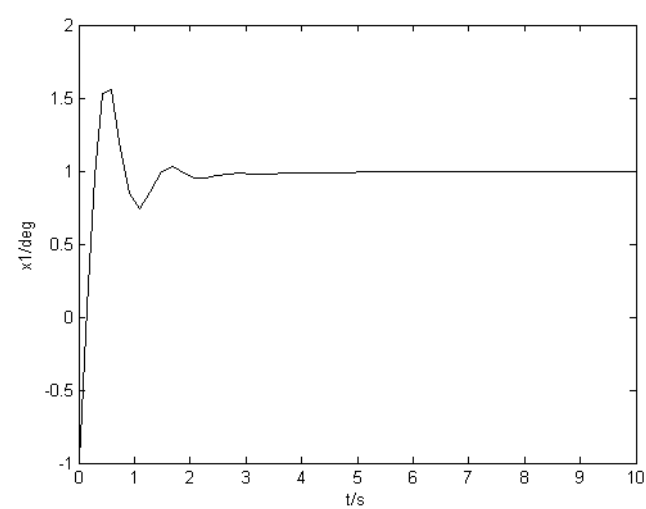

Figure 9. Result of Sixth Group

The above simulation result shows that the system can be unstable for some group of random parameters. So the PID control is not always effective.

\subsection{Sliding Synchronous Control}

According to above design method, parameters are choose as follows: $c_{1}=8, c_{2}=5, k_{1}=10, k_{2}=10, k_{3}=10, \Gamma_{1}=1, \Gamma_{2}=1, \Gamma_{3}=1$. Also choose six group of random number for matrix $\mathrm{A}$ and simulation results are shown as follows:

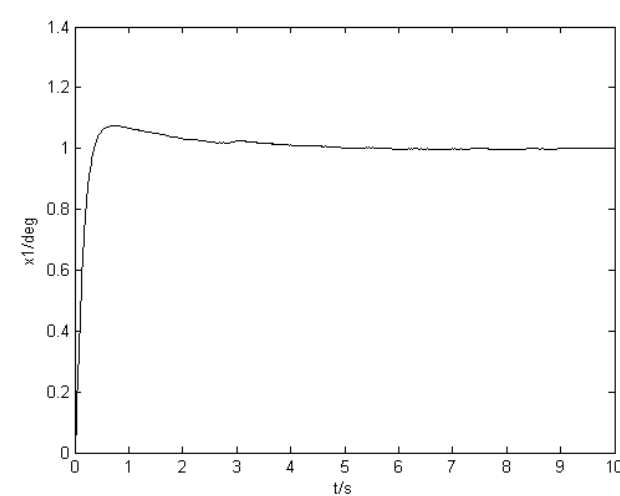

Figure 10. Result of First Group

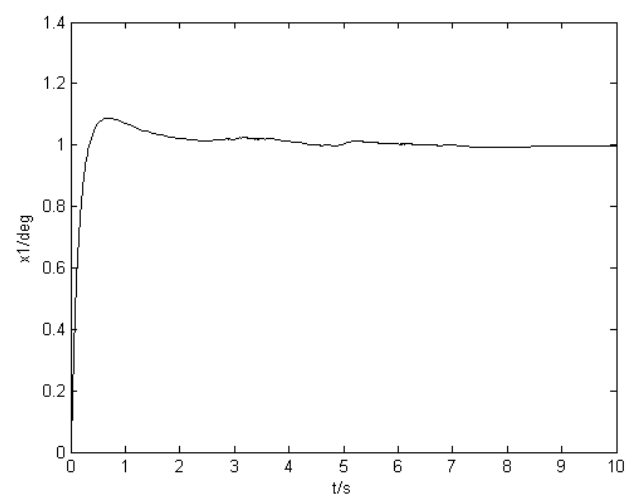

Figure 11. Result of Second Group 


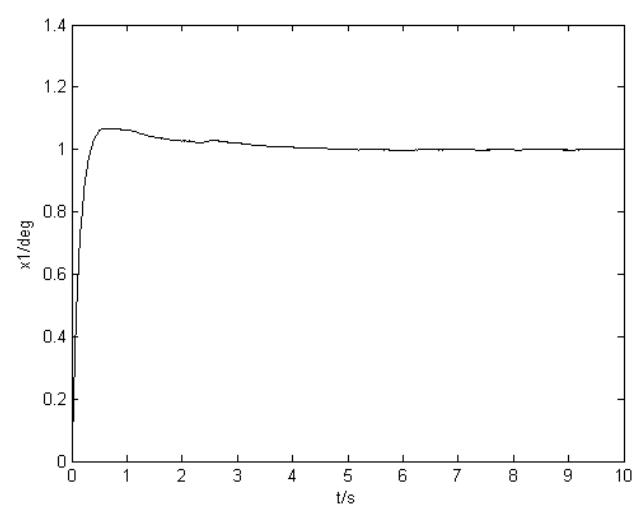

Figure 12. Result of Third Group

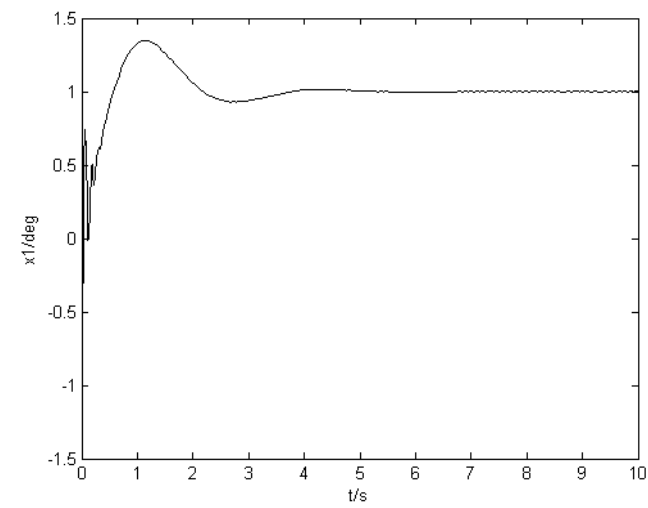

Figure 14. Result of Fifth Group

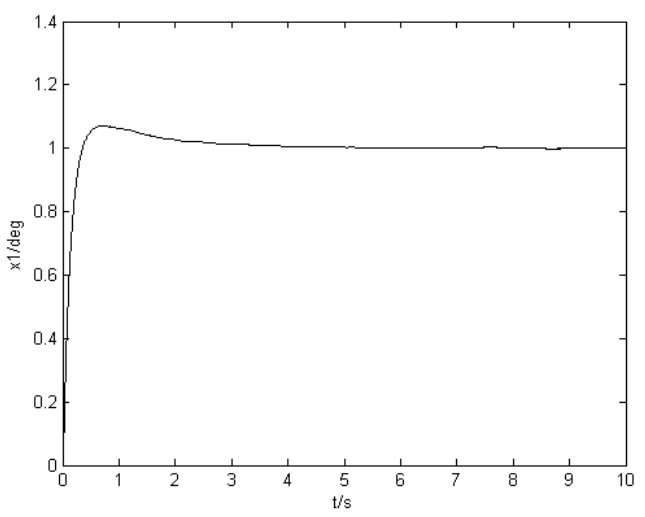

Figure 13. Result of Fourth Group

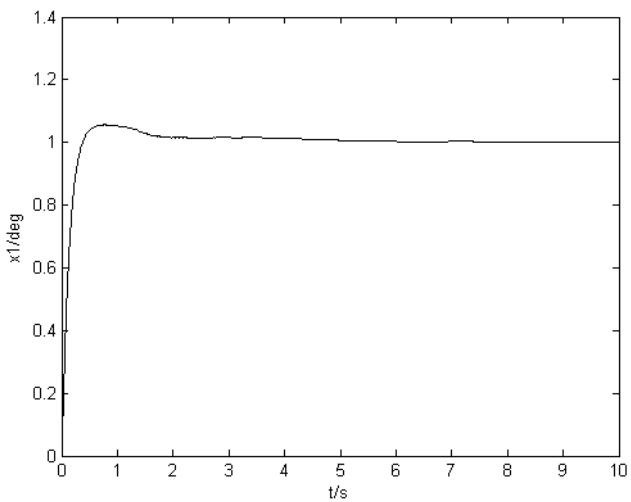

Figure 15. Result of Sixth Group

The conclusion can be made as follows: it is necessary to know parameters of control system in advance for the chose of parameters of PID controller. Especially, an optimal controller should be designed by transfer function of control object. So it is difficult to choose a proper PID controller for a group of random second order systems. And it is very convenient for the sliding mode synchronization method to solve the random systems by constructing a auxiliary system. Also the main advantage of the synchronous control method is that the exact model parameters are not necessary to be known at the beginning of the design.

\section{Conclusions}

A new kind of sliding synchronous control method which is mostly used in synchronization of communication system in the past research papers, is firstly proposed in this paper to cope random second order systems. Also it is compared with PID control and sliding mode control. Numerical simulation shows that the advantage of proposed method is that the parameters of control object are not necessary to be known in advance or even they can be random numbers of a given interval.

\section{Acknowledgment}

The author wish to thank his friend Heidi in Angels (a town of Canada) for her help, and thank his classmate Amado in for his many helpful suggestions. This paper is supported by Youth Foundation of Naval Aeronautical and Astronautical University of China, National Nature Science Foundation of Shandong Province of China 
ZR2012FQ010, National Nature Science Foundations of China 61174031, 61004002, 61102167, Aviation Science Foundation of China 20110184 and China Postdoctoral Foundation 20110490266.

\section{References}

[1] S. S. Ge, C. Wang and T. H. Lee, "Adaptive backstepping control of a class of chaotic systems", Int J Bifurcation and chaos, vol. 10, no. 5, (2000), pp. 1140-1156

[2] S. S. Ge and C. Wang, "Adaptive control of uncertain Chua's circuits", IEEE Trans Circuits System, vol. 47, no. 9, (2000), pp. 1397-1402

[3] L. Alexander, Fradkov and M. A Yu, "Adaptive synchronization of chaotic systems based on speed gradient method and classification", IEEE Trans Circuits System, vol. 44, no. 10, (1997), pp. 905-912

[4] X. Dong and L. Chen, "Adaptive control of the uncertain Duffing oscillator", Int J Bifurcation and chaos, vol. 7, no. 7, (1997), pp. 1651-1658

[5] T. Yang, C.-M. Yang and L.-B. Yang, "A Detailed Study of Adaptive Control of Chaotic Systems with Unknown Parameters", Dynamics and Control, no.8, (1998), pp. 255-267

[6] X. Yu, "Tracking inherent periodic orbits in chaotic dynamic systems via adaptive", IEEE Trans Circuits System, vol. 46, no. 11, (1999), pp. 1408-1411

[7] L. Chen, G. Chen and Y.-W. Lee, "Fuzzy modeling and adaptive control of uncertain chaotic systems", Information Sciences, vol. 121, no. 1, (1999), pp. 27-37

[8] L. Chen and G. Chen, "Fuzzy adaptive control of uncertain chaotic Systems using time", Int J Bifurcation and chaos, vol. 9, no. 40, (1999), pp. 757-767.

[9] C. T. Lin, "Controlling chaos by GA-based reinforcement learning neural network", IEEE Trans On neural networks, vol. 10, (1999), pp. 846-859

[10] J. Yan and C. Li, "On synchronization of three chaotic systems", Chaos, Solitons and Fracrals, no. 23, (2005), pp. 1683-1688.

[11] E.M. Elabbasy, H.N. Agiza and M.M. El-dessoky, "Adaptive synchronization of System with uncertain parameters", Chaos, Solitons and Fractals, no. 21, (2004), pp. 657-667.

[12] M.T. Yassen, "Chaos synchronization between two different chaotic systems using active control", Chaos, Solitons \& Fractals, vol. 23, no. 4, (2005), pp. 131-140.

[13] H.N. Agiza and M.T. Yassen, "Synchronization of Rossler and Chen chaotic dynamical systems using active control", Physics Letters A, no. 278, (2001), pp. 191-197.

[14] M.-C. Ho and Y.-C. Hung, "Synchronization of two different systems by Using generalized active control", Physics Letters A, no. 301, (2002), pp. 424-428.

[15] E.M. Elabbasy, H.N. Agiza and M.M. El-Dessoky, "Adaptive synchronization of a hyper chaotic system with uncertain parameter", Chaos, Solitons and Fractals, vol. 30, (2006), pp. 1133-1142.

[16] F. Tang and L. Wang, "An adaptive active control for the modified Chua's circuit", Physics Letters A, vol. 346, (2005), pp.342-346.

[17] M. Hu, Z. Xu, R. Zhang and A. Hu, "Parameters identification and adaptive full state hybrid projective synchronization of chaotic (hyper-chaotic) systems", Physics Letters A, vol. 361, (2007), pp.231-237

[18] Z.-M. Ge and C.-H. Yang, "Pragmatical generalized synchronization of chaotic Systems with uncertain parameters by adaptive control", Physica D, vol. 231, (2007), pp.87-89

[19] T. Gao, Z. Chen, Z. Yuan and D. Yu, "Adaptive synchronization of a new hyperchaotic system with uncertain parameters", Chaos, Solitons and Fractals, vol. 33, (2007), pp.922-928.

[20] X. Wang, J. Lei and C. Pan, "Trigonometric RBF neural robust controller design for a class of nonlinear system with linear input unmodeled dynamics", Applied Mathematics and Computation, 185, (2007), pp.989-1002.

[21] S.-H. Kim and Y.-S. Kim, "Chanho Song, A robust adaptive nonlinear control approach to missile autopilot design", Control engineering practice, vol. 12, (2004), pp. 149-154

[22] X. Wang and J. Lei, "Design of Global Identical Terminal Sliding Mode Controller for a Class of Chaotic System", 2006 IMACS Multiconference on Computational Engineering in Systems Applications (CESA'2006), Tsinghua University Press, vol. 10, (2006)

[23] J.W. Lei and Y. Wang, "Terminal sliding mode control of unit choatic system", Journal of Naval Aeronautical and Astronautical University, vol. 22, no. 3, (2007), pp. 309-312

[24] J. Lü, T. Zhou and S. Zhang, "Chaos synchronization between linearly coupled chaotic systems", Chaos, Solitons, Fractals, vol. 14, (2002), pp. 529-541.

[25] E. Ott, C. Grebogi and J.A. Yorke, "Controlling chaos", Phys. Rev. Lett., vol. 64, (1990), pp. 1196-1199.

[26] J.H. Park, "Adaptive synchronization of a unified chaotic systems with an uncertain parameter", Internat. J. Nonlinear Sci. Numer. Simulation, vol. 6, no. 2, (2005), pp. 201-206. 


\section{Author}

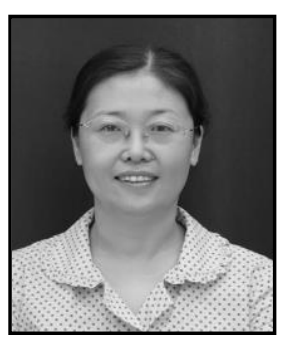

Xinyu Wang, she was born in Yantai, Shandong province of China in 1963. She received the B. Eng degree in Physics from Liaocheng Normal College in 1985. She received the Master Degree in System Science from Naval Aeronautical Astronautical University, Yantai of China in 2006. After that she continued her study there and received the Doctor degree in Communication and Information System in 2010. She worked as a teacher in Yantai University in Shandong province of China in 1985 and was promoted to be a vice professor in 1999 and became a professor in 2008. Now her present interests are chaotic system, communication, physics and information. 